\title{
Multiple Description Video Coding for Stereoscopic 3D
}

\author{
H. Abdul Karim, A. Sali, S. Worrall, Abdul H. Sadka, A. M. Kondoz
}

\begin{abstract}
In this paper, we propose an MDC schemes for stereoscopic $3 D$ video. In the literature, MDC has previously been applied in $2 D$ video but not so much in $3 D$ video. The proposed algorithm enhances the error resilience of the $3 D$ video using the combination of even and odd frame based MDC while retaining good temporal prediction efficiency for video over error-prone networks. Improvements are made to the original even and odd frame MDC scheme by adding a controllable amount of side information to improve frame interpolation at the decoder. The side information is also sent according to the video sequence motion for further improvement. The performance of the proposed algorithms is evaluated in error free and error prone environments especially for wireless channels. Simulation results show improved performance using the proposed MDC at high error rates compared to the single description coding (SDC) and the original even and odd frame MDC. ${ }^{1}$
\end{abstract}

Index Terms - 3D video, multiple description video coding, side information, error-resilience.

\section{INTRODUCTION}

Immersive media will be the next potential candidate in multimedia communication applications. The technological advancement of stereoscopic video capture, compression and display technologies enables the scaling of existing video applications into stereoscopic applications. 3D video allows users to feel the presence of the persons they are communicating with or be truly immersed in the event they are watching. 3D video is mainly being used in entertainment applications such as in cinema. To be able to have $3 \mathrm{D}$ video on consumer devices, a lot of research has been carried out on $3 \mathrm{D}$ video, with the aim of simple provision of $3 \mathrm{D}$ contents to users and of exploring the potential for $3 \mathrm{D}$ video communication [1] [2]. Over the years, many manufacturers have developed 3D displays that offer auto-stereoscopic 3D displays, allowing multiple users to view $3 \mathrm{D}$ content at the same time without 3D glasses [3]. 3D mobile phones are also

\footnotetext{
${ }^{1}$ This work was supported in part by VISNET II, a European Network of Excellence (http://www.visnet-noe.org), funded under the European Commission IST FP6 programme.

H. Abdul Karim is with the Multimedia University, 63100 Cyberjaya, Selangor, Malaysia (e-mail: hezerul@mmu.edu.my).

A. Sali is with the Putra University, 43400 Serdang, Selangor, Malaysia (e-mail: aduwati@eng.upm.edu.my).

S. Worrall is with the University of Surrey, Guildford, GU2 7XH UK (email: S.Worrall@surrey.ac.uk).

Abdul H. Sadka is with the Brunel University, Uxbridge, West London, UB8 3PHUK, (email: Abdul.Sadka@brunel.ac.uk)

A. M. Kondoz is with the University of Surrey, Guildford, GU2 7XH UK (e-mail: A.Kondoz@surrey.ac.uk).
}

Contributed Paper

Manuscript received August 10, 2009

0098 3063/09/\$20.00 @ 2009 IEEE being built, such as in [4], allowing communication in 3D.

When $3 \mathrm{D}$ video is compressed and transmitted over error prone channels, error propagation due to packet loss leads to poor visual quality. Hence, error resilience techniques for 3D video are needed. MDC is an effective way to combat burst packet losses in wireless and Internet networks. MDC is a promising approach for video application where retransmission is unacceptable [5]. MDC divides a source into two or more correlated layers. This means that a high-quality reconstruction is available when the received layers are combined and decoded, while a lower, but still acceptable quality reconstruction is achievable if only one of the layers is received. Hence, with $3 \mathrm{D}$ video $\mathrm{MDC}$, users can have a $3 \mathrm{D}$ visual communication system that is robust to packet losses.

Several MDC methods have been proposed in the literature. One of the most popular one is the multiple state video coding (MSVC) proposed in [6]. This method splits the input video into sequences of even and odd frames, each being coded as an independent description.

In this paper, the MSVC technique is used to produce the MDC for stereoscopic 3D video. Other MDC types are potentially more efficient, but MSVC is computationally simple, and standard compliant bit streams can be produced. It also introduces no mismatch when only one of the descriptions is received because the decoder uses the same prediction signal as the encoder for each generated description.

The rest of this paper is organized as follows. In this paper, A brief review of multiple description 2D video coding algorithms is presented in Section II, followed by the proposed MDC for stereoscopic 3D video in Section III. The performance of the algorithms is investigated through extensive simulation in error free and error prone channels in Section IV. The paper is finally concluded in Section V.

\section{OVERVIEW OF MULTIPLE DESCRIPTION CODING FOR 2D VIDEO}

MDC algorithms in the literature can be broadly categorised into three methods, MDC quantisation, MDC transform coding and MDC sub-sampling.

\section{A. Multiple Description Coding Method Based on Quantisation}

MDC quantisation splits the quantized coefficient into two or more streams. In a simple implementation of MDC quantisation algorithm, the multiple descriptions are produced by using two quantisers whose decision regions are offset by one-half of a quantisation interval of each other [8]. 
The MDC quantisation algorithm can be improved using embedded scalar quantiser, which can also achieve a fine grain scalable bit stream beside the error resilience [9]. The scalar quantiser will refine the source information successively using finer quantisers which can be created by further segmenting the steps of coarser quantiser [10]. Examples of MDC quantisation algorithms are in [11] and [12]. The proposed method in [11] is further enhanced in [12] by adding another quantiser in the central prediction loop.

\section{B. Multiple Description Coding Method Based on Transform Coding}

In MDC transform coding, the multiple descriptions in the form of transform coefficients are produced from the output of a transform coded block. In [13], pair-wise correlating transform (PCT) is proposed to transform a set of coefficients into two sets of correlated coefficients with controlled redundancy and side distortion. In [14], cascaded correlating transform as extension to the pair-wise correlating transform is proposed.

In wavelet transform based MDC such as in [15] and [16], the MDC streams are respectively produced from the arranged wavelet coefficients and the partitioned transform domain of the signals. Researchers in [17] investigated another wavelet based MDC, which produces multiple descriptions from the wavelet representation following a checker-board pattern. An MDC scheme and its application in multiple path transport have been investigated in [18]. Lapped orthogonal transform is used in the transform stage and the transformed coefficient is split into two descriptions using a checker-board pattern.

\section{Multiple Description Coding Method Based on Sub- Sampling}

In MDC sub-sampling, the original signal is decomposed into subsets, either in spatial, temporal or frequency domain, where each description corresponds to different subsets. This algorithm takes advantage of the correlation of the spatially or temporally adjacent video data samples. Examples of algorithms include temporal frame interleaving [6] and spatial pixel interleaving on image samples [19] or motion vectors using quincunx sub-sampling [20].

In [20] for example, the MDC streams are generated by encoding the motion vector field into two description using quincunx sub-sampling process. More on temporal frame interleaving MDC, specifically even and odd frames based MDC will be discussed in Section II-D as it is the basis of the proposed MDC techniques in this paper.

\section{Even and Odd Frames Based Multiple Description Coding}

Many even and odd frames based MDC methods are investigated in the literature due to its simplicity in producing multiple streams. The even and odd MDC basically includes the even and odd video frames into description one and two respectively [6]. An odd frame is predicted from previously reconstructed odd frame only as shown in Fig. 2. Prediction of the even frame is also similar to the odd frame. It is important to note that the two descriptions are independently coded so that when only a single stream is received at the decoder, it can be decoded with acceptable quality at lower temporal resolution. It also introduces no mismatch when only one of the descriptions is received because the decoder uses the same prediction signal as the encoder for each generated description. Compatibility with the existing video coding standard is another advantage for the even and odd frames MDC as the descriptions from this MDC can be decoded by the standard decoder, provided the descriptions are encoded using the standard encoder.

The redundancy in even and odd MDC comes from the longer temporal prediction distance compared to standard video coder, which uses the nearest past frame for prediction. Hence, its coding efficiency is reduced. This method is similar to the video redundancy coding (VRC) proposed in [21].

For a practical MDC scheme, it is necessary to control the redundancy to match the network conditions. The MDC method in [22] is similar to the VRC method, but the mismatch between the predicted frames at the encoder and decoder is also coded and appended in both descriptions. The predictor and the mismatch signal quantiser can be varied to control the redundancies. In [23], two streams of lowerresolution pictures are added to the multiple state video streams. In case one of the streams is lost, a spatial-temporal hybrid interpolation is used to recover the missing frames.

Performance of the multi-state video encoder proposed in [6] is improved by [24] using multi-hypothesis motion prediction at the encoder. Small additional block motion information is introduced, which helps fast error recovery at the decoder. Multi-state video encoder with side information is proposed in [25]. The side information, which is calculated offline at the encoder, will tell the decoder which reconstruction method will give optimal quality. This method outperforms the original multi-state encoder up to $1 \mathrm{~dB}$ depending on the loss rates of transmission channels.

All the MDC discussed before were applied to 2D video to provide error resilience. In case of 3D video, MDC schemes were proposed for 3D stereoscopic left and right views in [26] using spatial scaling and multi-state coding. Other MDC schemes which take advantage of encoding a source transmission over multiple channels have been used in [27]. This implores a novel MDC technique with side information for 3D video based on the even and odd frames. The redundant side information consists of the difference between the interpolated frame and the locally reconstructed frame that can be quantised, hence, the redundancies can be controlled by the quantisation parameter. This is then extended in [28] using Bi-directional frames (B-frame) coding technique to achieve reduced variable redundancies.

In [7] and [29], scalable MDC are proposed for the 2D plus depth format stereoscopic video. The method is then improved using motion interpolation and applied to $2 \mathrm{D}$ video in [30].

In this paper, the method in [27] is extended to improve the performance of stereoscopic video transmission in error free and error prone condition using adaptive side information. 


\section{PROPOSED MULTIPLE DESCRIPTION 3D VIDEO CODING}

\section{A. Even and odd $M D C(M D C-E O)$}

The general block diagram for even and odd frame based MDC (MDC-EO) for 3D video is shown in Fig. 1. It is built upon the existing MPEG-4 video coding standard that has Multiple Auxiliary Component (MAC) tools to support depth information. There are texture part that includes luminance (Y) and chrominance ( $\mathrm{U}$ and $\mathrm{V}$ ) components, and also depth part (also called alpha plane) for each macro block in an even/odd video frame. The even and odd frames are predicted from previous even and odd frame respectively as in multisate encoder [6]. The even and odd frames are encoded into streams 1 and 2 respectively.

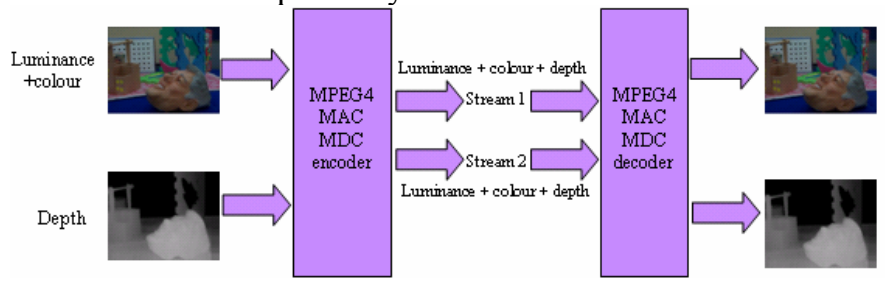

Fig. 1: The proposed MDC-EO encoder and decoder block diagram

The content of the two bit streams at the frame level for texture and depth information is shown in Fig. 2. Streams 1 and 2 contains even and odd frames respectively. The content of the two bit streams at the macro-block level for texture and depth information is shown in Fig. 3. The alpha information is actually the depth information.

If both even and odd streams are received, the decoder can reconstruct the coded sequence at full temporal resolution. If only one stream is received, the decoder can still decode the received stream at half the original temporal resolution. Since the even frames are predicted from previous even frames (independent from odd frames), there will be no mismatch if one of the streams are lost at the decoder.

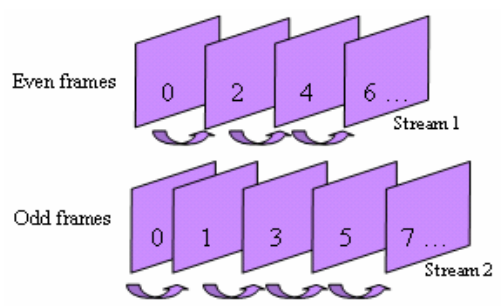

Fig. 2: Contents of stream 1 and 2 at frame level

Additionally, in the case of one stream is received, the decoder can decode at full resolution by interpolating between the received frames as in [6]. Frame interpolation is performed using (1).

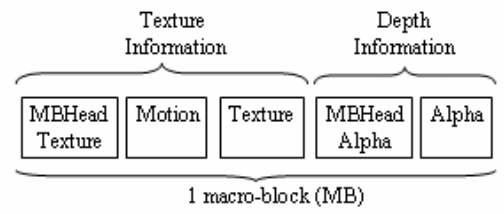

Fig. 3: Contents of stream at macro-block level

$$
I_{i p}(i, j)=\frac{I_{\text {prev }}(i, j)+I_{\text {fut }}(i, j)}{2}
$$

where $I_{i p}(i, j)$ is the frame to be interpolated at pixel location $(i, j), I_{\text {prev }}(i, j)$ is the previous frame and $I_{f u t}(i, j)$ is the future frame. This average frame interpolation is used in the simulation when there are errors in a frame. Motion compensated frame interpolation can also be used to obtain improved performance as in [6] but at the expense of decoder complexity.

The even and odd MDC is developed on top of the MPEG4-MAC codec. A frame buffer is used to store the previous ( $n$ $2)$ reconstructed frame, $F^{\prime}(n-2)$. If the input is an even frame, then the coded residual, $E c q(n)$ is appended into stream 1 and vice versa for the odd frame.

\section{B. Even and odd MDC with side information (MDC-EOS)}

MDC-EO in Section III-A performs better than SDC in a high error rate situation. If for example, one stream is corrupted, it can be replaced with the interpolated frames of the other stream provided that the other stream is not in error. The interpolation produces a blurred image, especially if the difference between the frames used in the interpolation is large as shown in the example in Fig. 4. It also produces large PSNR variation between frames when errors occur. The frame PSNR is low for the interpolated frame and high for the uncorrupted frame in the other stream. The frame PSNR for the following frames predicted from the interpolated frame are also affected by the error.

To reduce the PSNR variation and the blurring effect, it is proposed to send controllable side information on top of the MDC-EO at the expense of reduced coding efficiency in error

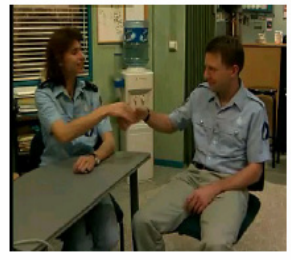

(a)

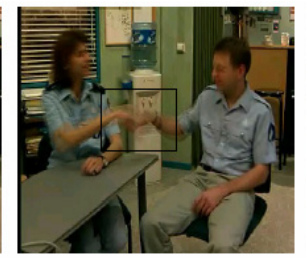

(b)

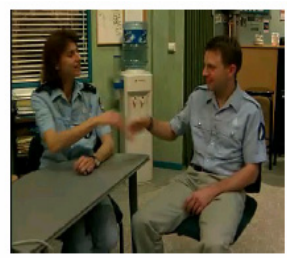

(c)
Fig. 4: Frame Interpolation (a) previous frame (b) blurred interpolated frame (especially in the highlighted box) (c) next frame

free environments. The block diagram for our proposed MDC for 3D video (MDC-EOS) is shown in Fig. 5 (encoder) and Fig. 6 (decoder). The even and odd frames are encoded into streams 1 and 2 respectively. Each frame contains texture, motion and depth data. Side information for even and odd stream frames is also appended to their corresponding streams.

At the encoder, the central encoder is used to produce even or odd frames. The frame buffer is used to store the reconstructed frames, $F^{\prime}(n-1)$ and $F^{\prime}(n-2)$. Even frames are predicted from previous reconstructed even frames and vice versa for odd frames.

Side encoder 1 and 2 are used to produce the side information for even and odd stream respectively. In side 
encoder 1, frame interpolation is performed between the current reconstructed even frame, $\mathrm{Fe}^{\prime}(\mathrm{n})$ and the previous reconstructed even frame, $\mathrm{Fe}^{\prime}(\mathrm{n}-2)$. Equation (1) is used to produce the interpolated frame. Only side encoder 1 is shown in Fig. 5, but side encoder 2 has similar structure.

The interpolated frame is subtracted from the previous reconstructed frame, $F^{\prime}(n-1)$ and the difference, $\mathrm{Ee}(n)$, which is the side information, is coded using DCT and quantisation. Hence, the redundancy introduced can be controlled by varying the quantisation parameter (Q1) of the side information.

At the decoder in Fig. 6, the central decoder is used to decode the central information (even or odd frames). If for example only an even stream is received, side decoder 1 is invoked to recover the odd frame, $F_{O}{ }^{\prime}(n)$. The results of frame interpolation of the previous reconstructed even frame $F_{c}$ ' $(n$ 2 ), and previous reconstructed frame $F_{c}{ }^{\prime}(n)$, is added to the decoded side information, $E e^{\prime}(n)$, to get $F_{O}{ }^{\prime}(n)$.

In this way, if the quantisation parameter of the side information (Q1) is low, a high quality interpolated frame is produced at the decoder at the expense of higher redundancies. On the other hand, if Q1 is high, a reduced quality interpolated frame is produced but at lower redundancies. These features allow us to control the amount of redundancies needed for the MDC coder. These operations are extended to include the depth data. The content of the two bit streams at the macro block level for texture and depth information is shown in Fig. 7(a). The content of the two bit streams at the macro block level for the side information is shown in Fig. 7(b).

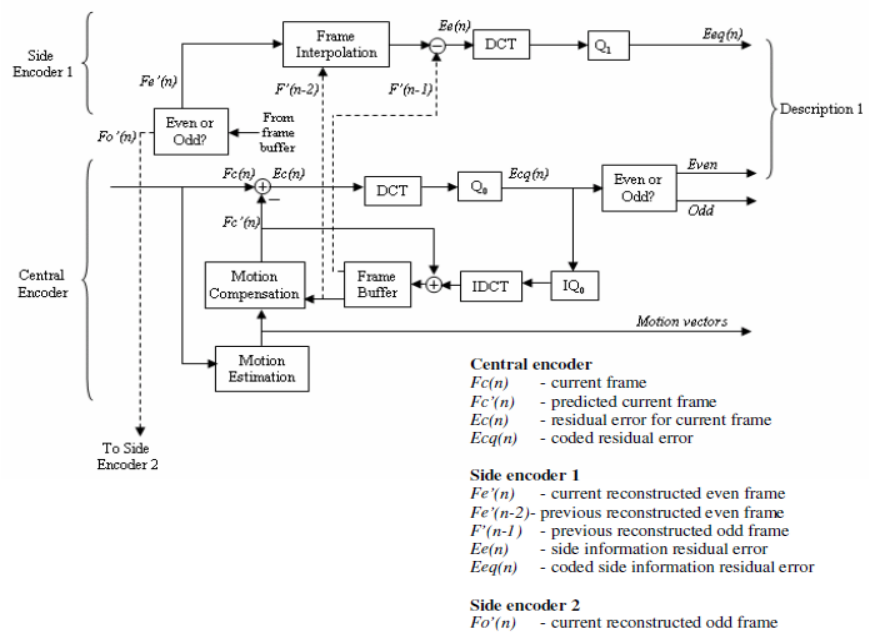

Fig. 5. Block diagram of the proposed MDC-EOS encoder

It is mentioned in [8] that one of the redundancies in a predictive multiple description video coder is any bit-rate used to describe side information in excess of that used by an SDC. For the MDC-EOS method, this extra signal is called Ee(n) (Eon $(n)$ for odd frame), which is the difference between the reconstructed and the interpolated frames. $E e(n)$ is used to reconstruct the odd frames when only even frames are received. However, in error free conditions, $\mathrm{Ee}(n)$ is not used.

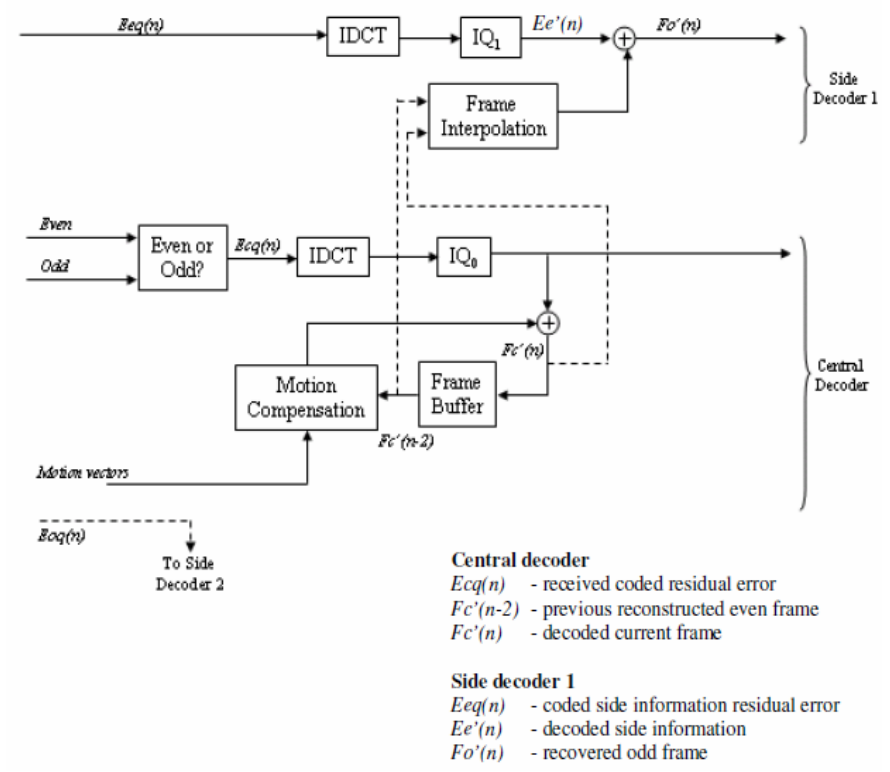

Fig. 6. Block diagram of the proposed MDC-EOS decoder

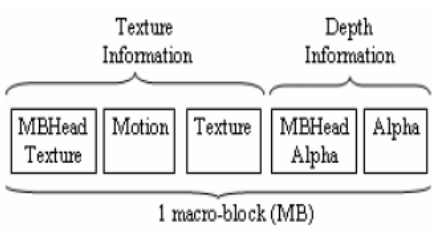

(a)

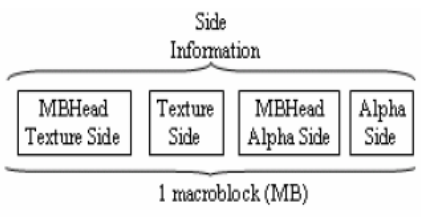

(b)
Fig. 7. Content of bit stream at macro-block level for (a) Central information and (b) Side information

In other words, this side information is ignored when both descriptions are received, similar to [22]. Hence, it is proposed in Section III-C to use $E e(n)$ in error free conditions.

\section{Even and odd MDC with side information and prediction (MDC-EOSP)}

In both MDC-EO and MDC-EOS method, frame $n$ is predicted from frame $n-2$, causing a decrease in coding efficiency in the central prediction due the usage of predictor that is less efficient than the SDC predictor (in SDC, frame $n$ is predicted from frame $n-1)$. Hence we proposed to use the side information, $E e(n)$, to improve the central prediction in error free conditions.

The detailed block diagram of MDC-EOSP is shown in Fig. 8. Compared to MDC-EOS, there is a new block called $P$ in the central encoder. The decoded $E e(n)$ is added to the interpolated frame to obtain Fip' and is used for the prediction of frame $n$. Using the idea of multiple predictions as in [22], frame $n$ is predicted from the superposition of Fip' and $F^{\prime}(n-$ 2) frame.

For $n>=4, n$ is predicted from $\mathrm{P}$, which is defined in (2),

$$
\mathbf{P}=a_{1} F_{i p}{ }^{\prime}+a_{2} F^{\prime}(n-2)
$$

where $a_{1}$ and $a_{2}$ are the weighting factors for $F_{i p}$ ' and $F^{\prime}(n-2)$ respectively. $F_{i p}$ ' is the interpolation of frames $n-2$ and 


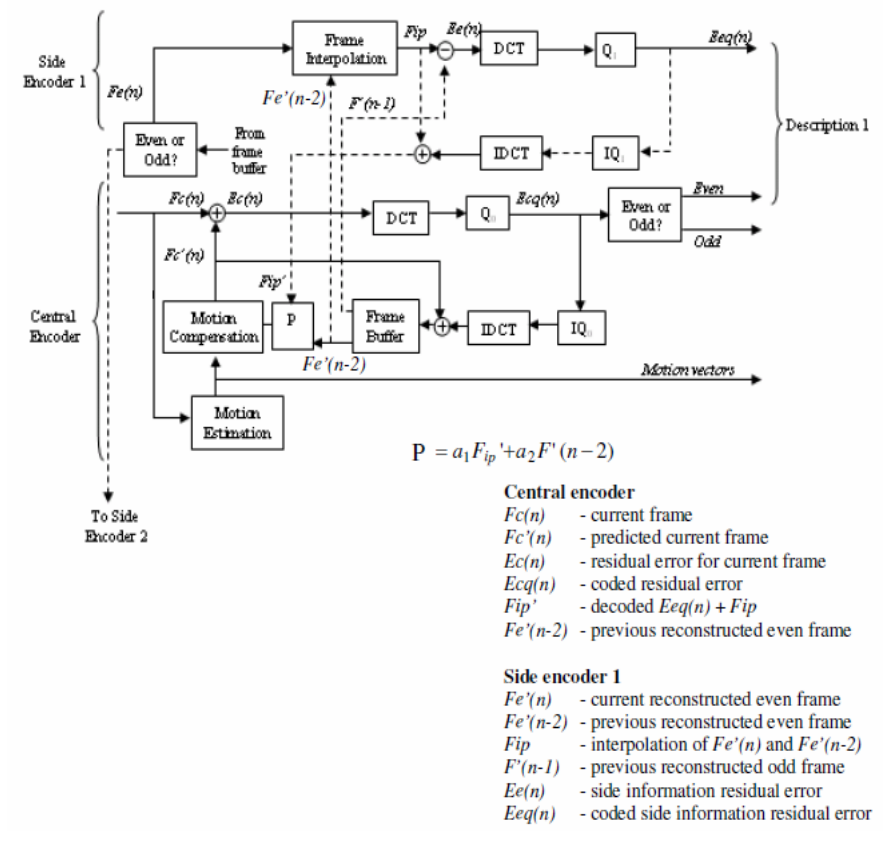

Fig. 8. Block diagram of the proposed MDC-EOSP encoder

frames $n-4$ plus the difference of the interpolated frame and the reconstructed odd frame. In (2), $F^{\prime}(n-2)$ is $F e^{\prime}(n-2)$ if the current frame is an even frame.

The sum of $a_{1}$ and $a_{2}$ must be equal to 1 following the approach of leaky predictor in [22]. Note that $F_{i p}$ ' is equal to the reconstructed $n-3$ frame if quantisation and inverse quantisation block are absent. Basically, for $n=4$, the prediction comes from a weighted sum of reconstructed frames $n-3$ and $n-2$. The prediction is applied to frame $n>=4$ because the interpolated frame of $n-3$ is available only from $n=4$. As an example, for $n=3$ frame, the interpolated frame is frame $n=0$, which is not available. $a_{1}$ and $a_{2}$ values can be adjusted to provide different weighted sums of prediction. It is found from experiments, that $a_{1}=0.1$ and $a_{2}=0.9$ gives the best result in terms of PSNR and total bit rate, which means more weight to frame $n-2$.

The side encoder section performs frame interpolation between the current even frame, $\mathrm{Fe}(n)$, and the previous even frame, $F e^{\prime}(n-2)$, to produce Fip. The difference between the interpolated frame, Fip, and the previous reconstructed frame (or the odd frame), $F^{\prime}(n-1)$, is coded using DCT and Q1 (side information quantiser) to produce $E e q(n)$. Decoded Eeq $(n)$ is added back to Fip to form Fip'. Ideally, Ee(n) should be added back to Fip, but to avoid mismatch prediction at the decoder, decoded $E e q(n)$ is used. In other words, $E e(n)$ is not available at the decoder, but decoded $E e q(n)$ is available to be added to Fip.

The difference between this method and [22] is block P which is located before the motion compensation process. Also with the proposed configuration, there is no motion vector sent as side information, and no mismatch signal needs to be coded as the even and odd frames are separately predicted.
Application of the proposed method to existing video coder will involve minimal addition of side encoder and decoder for the purpose of frame interpolation. The frame interpolation block only requires simple addition and division. The central encoder will require memory of previous $n-2$ frame which is made available by the current video coding standard such as H.264.

\section{Even and odd MDC with adaptive side information}

It was found that MDC-EOS and MDC-EOSP have reduced coding efficiency due to the large redundancies in the side information. Hence, it is proposed in this section to send the side information adaptively according to the motion in the sequence. If the motion is larger than a threshold, side information is appended to the bit stream. If the motion is smaller than the threshold, no side information is sent. This is because interpolation does not cause much degradation at low motion.

A method in [31] is used to estimate the amount of motion between frames. It exploits the data partitioning mode of MPEG-4 that placed the motion in first partition of the video packet. A value named ' $A$ ', which is the proportion of the video packet size occupied by the first partition, can be related to the amount of motion. 'A' can be expressed as:

$$
A=\frac{Y_{M B}}{X_{M B}+Y_{M B}}
$$

where $Y_{M B}$ is the average number of bits per macro block in the first partition, and $X_{M B}$ is the average number of bits per macro block in the second partition. Fig. 9 shows the variation in 'A' over the Interview sequence used in this paper for 300 frames. The period of high motion can be detected through the large values in ' $A$ '. In the Interview sequence for example, this period is after about frame 70 when the two subjects shake their hand.

The side information for MDC-EOS and MDC-EOSP is then sent according to this ' $\mathrm{A}$ ' value. The MDC-EOS and MDC-EOSP are now known as MDC-EOAS and MDCEOASP respectively. If the value of ' $A$ ' is bigger than a predetermined threshold, then the side information is sent. The threshold value is determined from Fig. 9. It was selected so that only minimum needed amount of side information is sent. For Orbi sequence the threshold value is set to 0.34 and for Interview threshold value is 0.15 .

\section{Simulation Results AND Discussion}

\section{A. Error free environment}

In order to evaluate the coding performance of the encoder in error free environments, we plotted a rate distortion curve for the Orbi sequence. The tests are carried out using CIF format (352x288). The basic encoding parameters are: 300 frames, IPPP... sequence format (only the first frame is encoded as an Iframe and all others are encoded as P-frames), 30 frames/s original frame rate, variable length coding (VLC) and without 


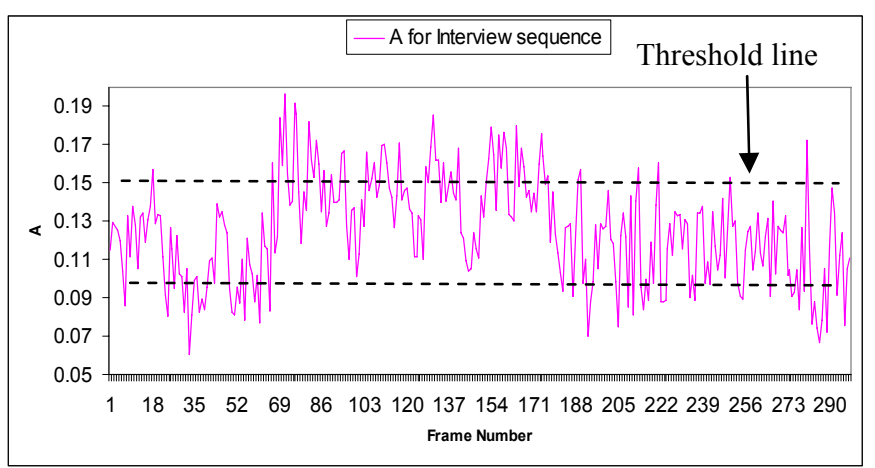

Fig. 9. Variation of $A$, the proportion of a packet occupied by the first partition, over the Interview sequence

error resilience. The quantisation parameter (QP) in the configuration file is varied to obtain the bit rate range shown in the rate-distortion curves. The rate distortion curves show the image quality measured in PSNR (PeakSignal-to-Noise Ratio) against the resulting bit rate when both of the MDC streams are received in error free, also known as central distortion.

Fig. 10 shows the rate-distortion curves for Orbi colour and depth sequences for SDC, MDC-EO, MDCEOAS and MDC-EOASP. For MDC-EOAS and MDCEOASP, the quantisation parameter for the side information is set to 20 . The rate distortion curve is quite close to MDC-EO because most of the side information is not sent as it is below the threshold. Hence, more bits are available to send the central information using a lower quantisation parameter.

Fig. 11 and Fig. 12 show the improvement obtained by MDC-EOAS and MDC-EOASP respectively for the luminance only. Same improvement is obtained with the depth information. At the same bit rate, the MDC algorithms with adaptive side information are about 1 to 2 $\mathrm{dB}$ better than without the adaptive side information.

\section{B. Same Bit Rate Experiment in Error Prone Environment}

The compressed 3D video is transmitted over a simulated wireless LAN channel. The WLAN error patterns used in this paper are obtained from the simulated WLAN channel described in [32].

The system parameters for the WLAN IEEE802.11g are: 1) Carrier Modulation: OFDM, 2) FFT Size: 64, 3) Carrier Frequency: $2.4 \mathrm{GHz}, 4)$ Sampling Rate: $20 \mathrm{MHz}$, 5) Channel Coding : Punctured Convolutional Coding. The combination of channel coding and modulation schemes produces several transmission modes with different data rate as up to $54 \mathrm{Mbit} / \mathrm{s}$. Several channel models are adopted with different environments and delay spreads. Rayleigh fading mobile channel is used and the environment characteristics include small office, medium office, large office and outdoor with or without LOS.

If an error occurs in a frame of one stream of the MDCEOAS and MDC-EOASP algorithm, the frame is replaced by the interpolated frame from the other stream plus the adaptively received side information. In this section the side information may be corrupted by the error. The QP used in the simulation for SDC, MDC-EO, MDC-EOAS and MDC-EOASP to achieve $512 \mathrm{kbit} / \mathrm{s}$ and its corresponding error free PSNR is shown in Table I for Interview sequence.

TABLE I.

QUANTISATION PARAMETER FOR INTERVIEW SEQUENCE AND THE CORRESPONDING ERROR FREE PSNR

\begin{tabular}{|l|c|c|c|c|c|c|c|}
\hline Encoder & \multicolumn{2}{|c|}{ Texture } & $\begin{array}{c}\text { Average } \\
\text { PSNR }\end{array}$ & \multicolumn{2}{c|}{ Depth } & $\begin{array}{c}\text { Average } \\
\text { PSNR }\end{array}$ & $\begin{array}{c}\text { QP } \\
\text { Side }\end{array}$ \\
\hline Frame & I & P & & I & P & & \\
\hline SDC & 12 & 7 & 35.30 & 13 & 8 & 38.21 & N/A \\
\hline MDC-EO & 5 & 9 & 34.16 & 8 & 12 & 35.83 & N/A \\
\hline MDC-EOAS & 3 & 10 & 33.99 & 6 & 15 & 34.98 & 15 \\
\hline MDC-EOASP & 4 & 10 & 33.99 & 4 & 16 & 35.14 & 15 \\
\hline
\end{tabular}

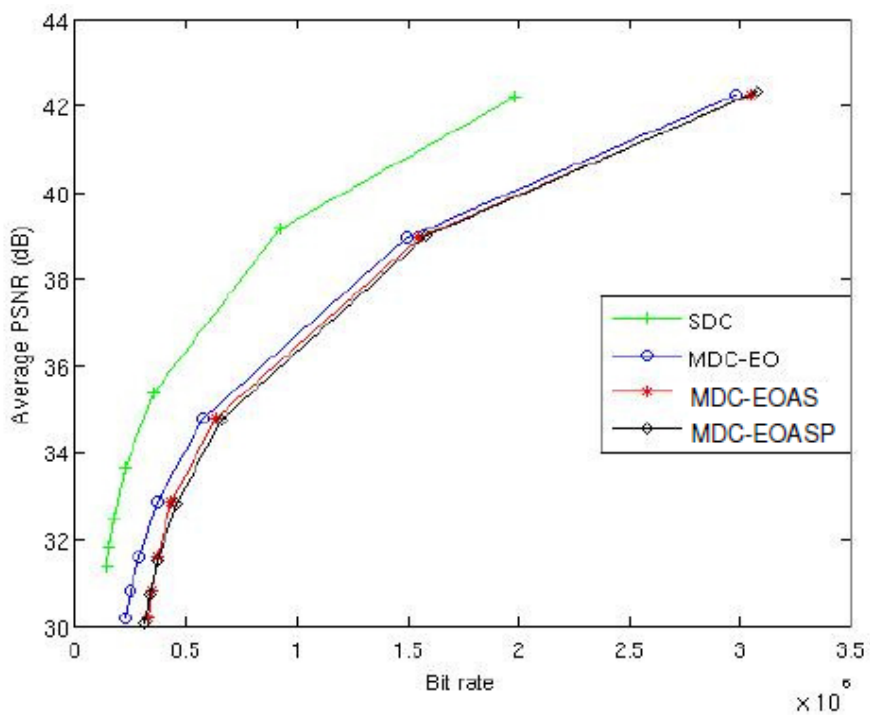

(a)

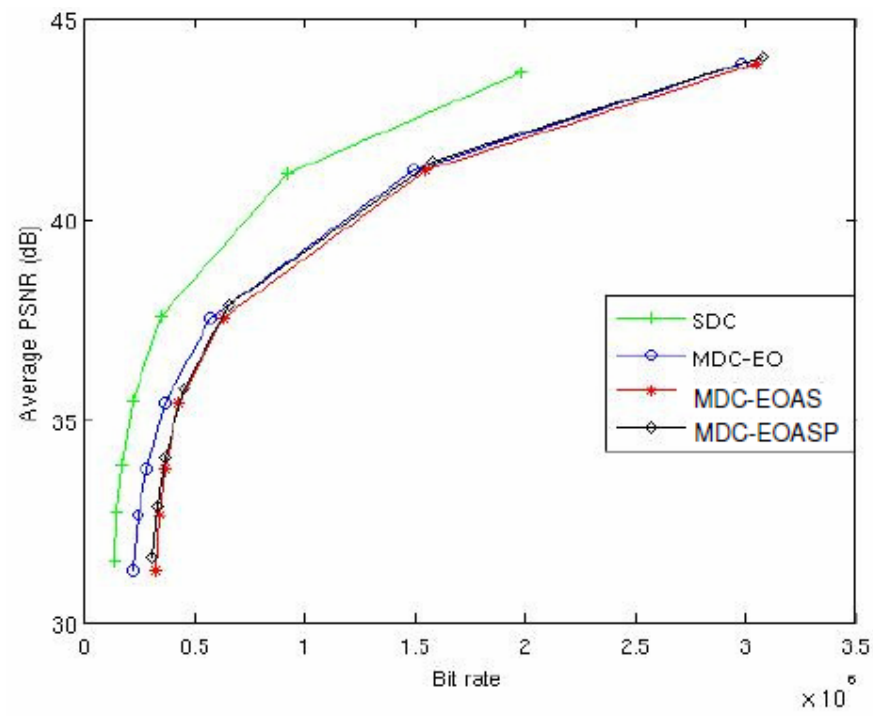

(b)

Fig. 10. Rate-Distortion curves for 'Orbi' sequence (a) Colour image sequence (b) Depth image sequence 


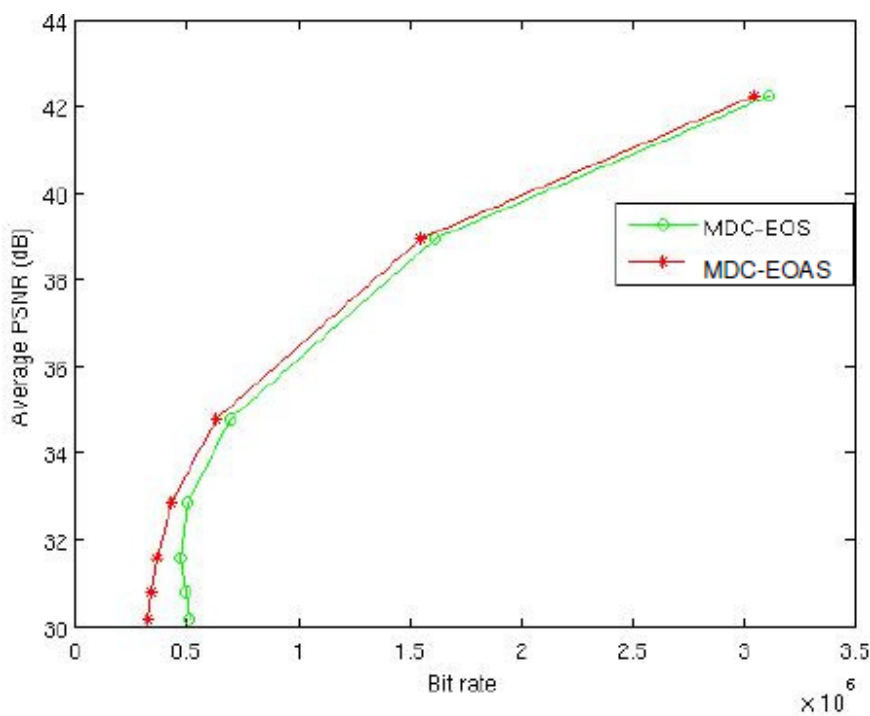

Fig.11. Rate-Distortion curves for MDC-EOS and MDC-EOAS for the luminance of 'Orbi' sequence

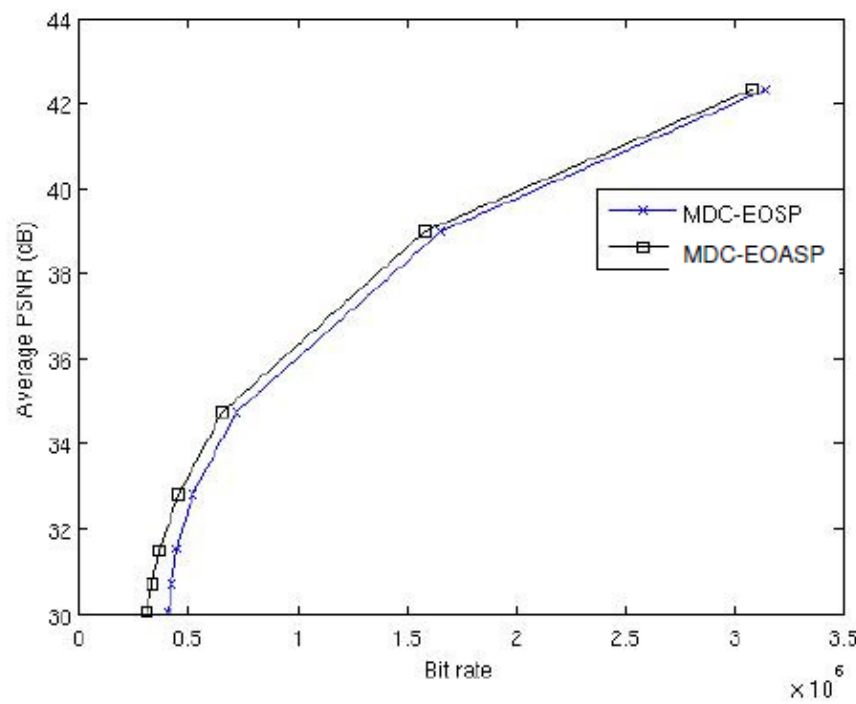

Fig.12. Rate-Distortion curves for MDC-EOSP and MDC-EOASP for the luminance of 'Orbi' sequence

Fig. 13 shows the results for the experiments for the Interview sequence. From the mean PSNR results, it can be seen that for the Interview sequence, MDC-EOASP result is comparable to MDC-EOAS for luminance and slightly better for depth. MDC-EOAS and MDC-EOASP is also better than SDC and MDC-EO at packet loss above $10 \%$. At $20 \%$ packet loss, MDC-EOASP mean PSNR is about $0.5 \mathrm{~dB}$ better than SDC for luminance and about $3 \mathrm{~dB}$ better than SDC for depth.

The small gain in luminance achieved by MDCs algorithms in error prone environment is probably due to the corruption of both MDC streams at the same time, which, violate MDC assumptions. Nevertheless, more gain is achieved in depth than luminance. Due to its content, the corrupted frame for depth data that is concealed or replaced using frame interpolation and the side information in MDCEOASP is better than corrupted macro block in a frame of SDC that was replaced with the corresponding macro block in

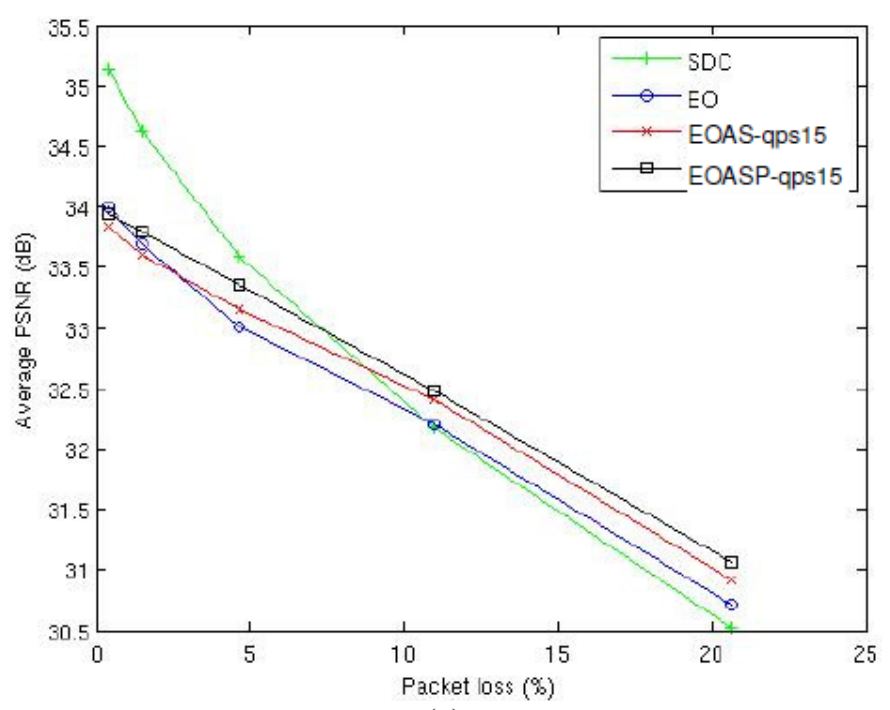

(a)

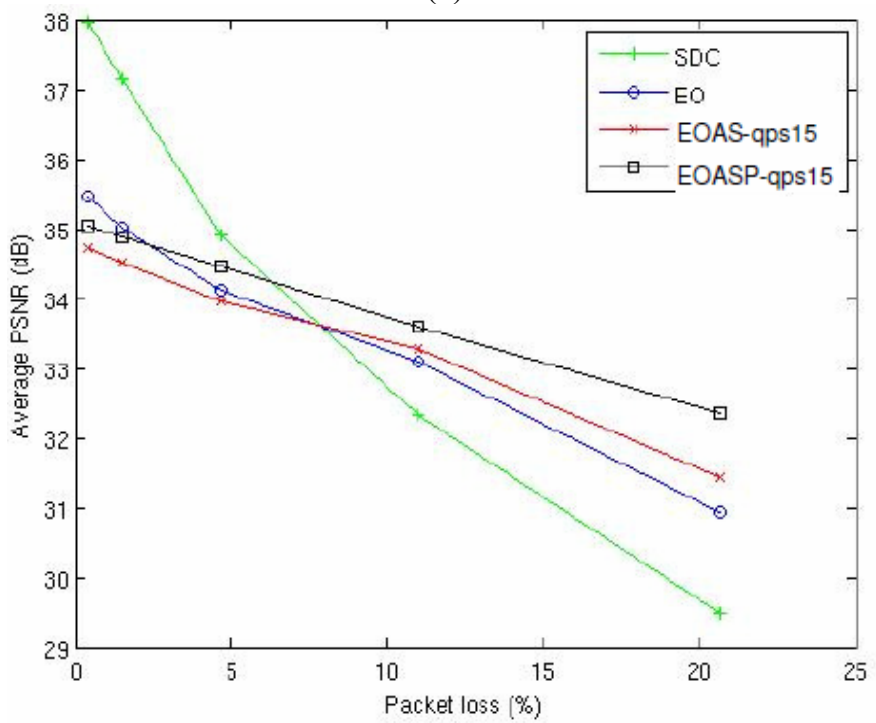

(b)

Fig. 13. Mean PSNR vs packet loss for Interview (a) luminance and (b) depth

the previous frame. This factor makes the average PSNR of MDC-EOASP is largely better than SDC for depth information, but slightly better than SDC for luminance information in high error rates.

Error free performance of MDC-EOAS and MDC-EOASP are comparable to MDC-EO because their coding efficiency is quite close as the side information is adaptively sent to the decoder. A similar pattern of results can be observed in the Orbi sequence [33].

The luminance subjective quality of frame 78 for the Interview sequence when subjected to $20 \%$ packet loss is shown in Fig. 14. The luminance PSNR for that frame is $26.79 \mathrm{~dB}, 28.54 \mathrm{~dB}, 31.17 \mathrm{~dB}$ and $31.33 \mathrm{~dB}$ for SDC, MDCEO, MDC-EOAS and MDC-EOASP respectively. The depth frame PSNR for Fig. 15 is $24.24 \mathrm{~dB}, 27.47 \mathrm{~dB}, 32.18 \mathrm{~dB}$ and $32.10 \mathrm{~dB}$ for SDC, MDC-EO, MDC-EOAS and MDCEOASP respectively. The 3D stereoscopic video quality can be obtained from the combination of the luminance, colour and depth as in [7]. Fig. 16 shows an improved 3D 
stereoscopic video quality with MDC-EOAS and MDCEOASP. The improvement, especially on the depth information, is achieved at the expense of side information generation and transmission. Fig. 16 can be viewed using a red and blue glass.

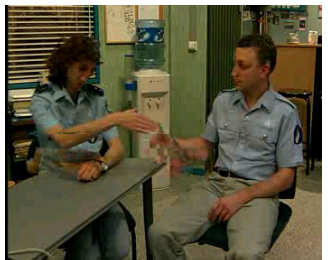

(a)

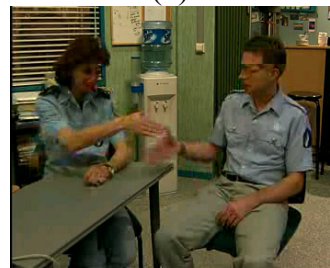

(c)

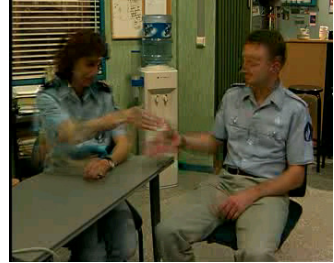

(b)

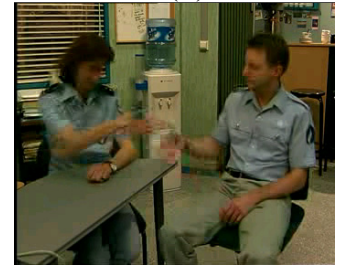

(d)
Fig. 14. Subjective quality - Interview - at $20 \%$ packet loss of luminance for (a) SDC and (b) MDC-EO (c) MDC-EOAS and (d) MDC-EOASP

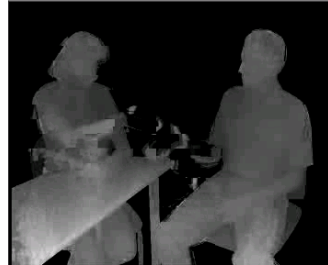

(a)

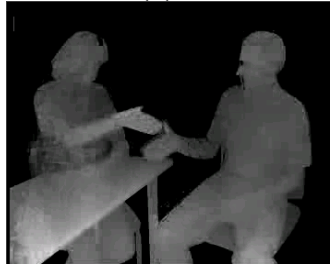

(c)

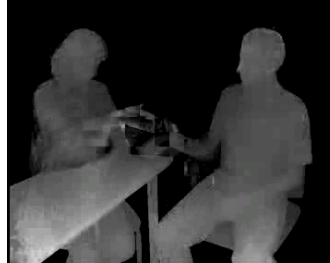

(b)

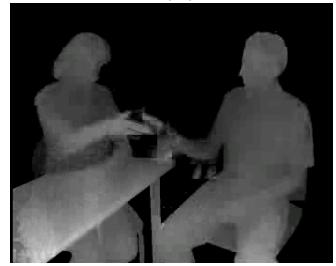

(d)
Fig. 15. Subjective quality - Interview - at $\mathbf{2 0 \%}$ packet loss of depth for (a) SDC and (b) MDC-EO (c) MDC-EOAS and (d) MDC-EOASP

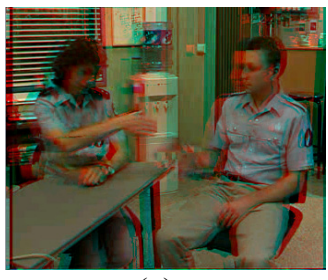

(a)

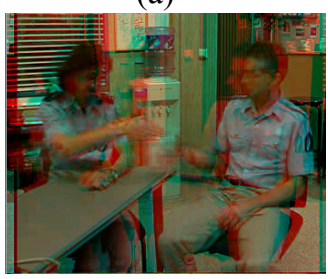

(c)

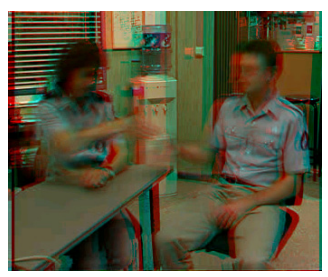

(b)

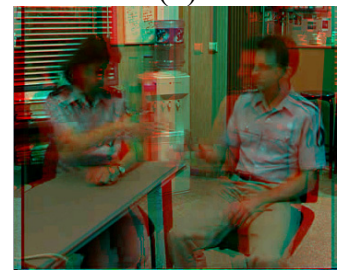

(d)
Fig. 16. Subjective quality - Interview - at $20 \%$ packet loss of stereoscopic 3D video for (a) SDC (b) MDC-EO (c) MDC-EOAS (b) MDC-EOASP

\section{CONCLUSION}

In this paper, we proposed MDC-EOS and MDC-EOSP for stereoscopic 3D video application. The side information in MDC-EOS and MDC-EOSP contributes to the high redundancy of these algorithms hence decrease in coding efficiency. We have also proposed a novel MDC-EOAS and MDC-EOASP to enhance error resilience by sending the adaptive side information. The side information is sent adaptively according to the motion in the sequence. Large motion will make the algorithm sends the side information at low motion no side information is sent. The coding efficiency of these two algorithms is better than MDC-EOS and MDCEOSP and very close to MDC-EO. The error prone performance of MDC-EOAS and MDC-EOASP is better than SDC and MDC-EO at high packet loss objectively and subjectively. The gain achieved by MDC-EOAS and MDCEOASP for depth is larger than the gain achieved for luminance. As a conclusion, MDC with side information is promising approach to combat channel errors for stereoscopic 3D video transmission, but the side information should be carefully sent as it can cause huge redundancies. It can be sent adaptively according to motion, and network conditions.

\section{ACKNOWLEDGMENT}

The work presented was developed within VISNET II, a European Network of Excellence (http://www.visnet-noe.org), funded under the European Commission IST FP6 program.

\section{REFERENCES}

[1] A. Redert, M. O. d. Beeck, C. Fehn, W. Ijsselsteijn, M. Pollefeys, L. V. Gool, E. Ofek, I. Sexton and P. Surman, "ATTEST: Advanced threedimensional television system technologies," in proc. of the 1st Int. Symp. on 3D Data Processing and Transmission (3DPVT'02), Padova, Italy, June 2002.

[2] http://www.3dtv-research.org/, 3DTV NoE, Last visited: March 2009.

[3] A. Sullivan, "3-deep," IEEE Spectrum, vol. 42, no. 4, pp. 22-27, April 2005.

[4] http://www.3dphone.org/, 3DPHONE-Project, Last visited: March 2009.

[5] Y. Wang, A. R. Reibman and S. Lin, "Multiple description coding for video communications," IEEE Proc., vol. 93, no. 1, pp. 57-70, January 2005.

[6] J. G. Apostolopoulos, "Error-resilient video compression via multiple state streams," in Proc. of Int. Workshop on Very Low Bit rate Video Coding, VLBV99, Kyoto, Japan, October 1999.

[7] H.A. Karim, C. Hewage, S. Worrall, A. Kondoz, "Scalable multiple description video coding for stereoscopic 3D", IEEE Trans. Consumer Electronics, vol. 54, No. 2, pp. 745 - 752, May 2008.

[8] V.K. Goyal, "Multiple description coding: Compression meets network", IEEE Signal Processing Magazine, vol. 18, no. 5, pp. 74-93, September 2001.

[9] F. Verdicchio, A. Munteanu, A. L. Gavrilescu, J. Cornelis and P. Schelkens, "Embedded multiple description coding of video", in Proc. of IEEE International Conf. on Image Processing (ICIP 2006), vol. 15, No. 10 , pp. $3114-3130$, Oct. 2006.

[10] http://www.freepatentsonline.com/20050254719.html, Embedded scalar quantisers with arbitrary dead-zone ratios - Patent 20050254719, Last visited: 3 June 2008.

[11] A. R. Reibman, H. Jafarkhani, Y. Wang, M. T. Orchard and R. Puri, "Multiple-description video coding using motion-compensated temporal prediction", IEEE Trans. Circ. and Sys. for Video Technology, vol. 12, no. 3, pp. 193-294, March 2002. 
[12] Y. C. Lee, Y. Altunbasak and R. M. Mersereau, "An enhanced two-stage multiple description video coder with drift reduction", IEEE Trans. Circ. and Sys. for Video Technology, vol. 14, no. 1, pp. 122-127, January 2004.

[13] Y. Wang, M. T. Orchard, V. Vaishampayan and A. Reibman, "Multiple description coding using pairwise correlating transforms", IEEE Trans. Image Processing, vol. 10, no. 3, pp. 351-366, March 2001.

[14] R. Knoerig and T. Sikora, "MDC image coding using cascaded correlating transform", in Proc. of Multimedia on Mobile Devices, IS\&T/SPIE $19^{\text {th }}$ Annual Symp. Electronic Imaging, CDROM, California, USA, January 2007.

[15] S. Somasundaram and K. P. Subbalakshmi, "3-d multiple description video coding for packet switched networks", in Proc. of Int. Conf. on Multimedia and Expo, vol. 1, pp. 589-592, July 2003.

[16] I. V. Bajic and J. Woods, "Domain-based multiple description coding of images and video", IEEE Trans. Image Processing, vol. 12, no. 10, pp. 1211-1225, October 2003

[17] N. V. Boulgouris, K. E. Zachariadis, A.N. Leontaris and M. G. Strintzis, "Drift-free multiple description video coding", in Proc. of IEEE Workshop on Multimedia Signal Processing, pp. 4034 - 4037, France, October 2001.

[18] N. Gogate, D. M. Chung, S. S. Panwar and Y. Wang, "Supporting image and video applications in a multihop radio environment using path diversity and multiple description coding", IEEE Trans. Circ. and Sys. for Video Technology, vol. 12, no. 9, pp. 777-792, September 2002.

[19] N. Franchi, M. Fumagalli, G. Gatti and R. Lancini, "A novel error-resilience scheme for a 3-d multiple description video coder", in Proc. of Picture Coding Symposium, PCS04, pp. 373-376, CA, USA, December 2004.

[20] C. S. Kim and S. U. Lee, "Multiple description coding of motion fields for robust video transmission", IEEE Trans. Circ. and Sys. for Video Technology, vol. 11, no. 9, pp. 999-1010, September 2001.

[21] S. Wenger, "Video redundancy coding in H.263+", in Proc. of Workshop on Audio-Visual Services for Packet Networks, Aberdeen, Scotland, September 1997.

[22] Y. Wang and S. Lin, "Error-resilient video voding using multiple description motion compensation", IEEE Trans. Circ. and Sys. for Video Technology, vol. 12 , no. 6, pp. 438-452, June 2002

[23] M-T. Lu, C-K. Lin, J. Yao and H. Chen, "Multiple description coding with spatial-temporal hybrid interpolation for video streaming in peer-topeer networks", Journal of Zhejiang University Science B, Vol. 7, no. 5, pp. 894-899, April 2006.

[24] G. Zhang and R. L. Stevenson, "Efficient error recovery for multiple description video coding", in Proc. of IEEE Int. Conf. on Image Processing, pp. 829-832, October 2004.

[25] S. Ekmekci and T. Sikora, "Multi-state video coding with side information", in Proc. of Asilomar Conference on Signals, Systems and Computers 2005, pp. 874-878, Oct. 28 - Nov. 12005.

[26] A. Norkin, A. Aksay, C. Bilen, G. Bozdagi Akar, A. Gotchev and J. Astola, "Schemes for multiple description coding of stereoscopic video," in Proc. LNCS, Mult. Content, Representation and Security, vol. 4105, pp. 730-737, Istanbul, Turkey, Sept. 2006.

[27] H. A. Karim, A. H. Sadka, S. Worrall and A. M. Kondoz ,"3D Video Error Resilience Using Multiple Description Coding", 2nd Workshop On Immersive Communication And Broadcast Systems, (ICOB 2005), Berlin, Germany, October 2005.

[28] H. A. Karim, A. H. Sadka, S. Worrall and A. M. Kondoz, "3D video error resilience using B-frame multiple description coding", in proc of Source Coding and Reliable Delivery of Multimedia Contents, Department 5 of NEWCOM, Toulouse, pp. 21-24, May 2006.

[29] H. A. Karim, C. T. E. R. Hewage, A. C. Yu, S. Worrall, S. Dogan and A. M. Kondoz, "Scalable multiple description 3D video coding based on even and odd frame", Picture Coding Symposium 2007, Lisbon, Portugal, November 2007.

[30] S. Adedoyin, W.A.C. Fernando, H.A. Karim, C.T.E.R. Hewage, A.M. Kondoz, "Scalable Multiple Description Coding with Side Information Using Motion Interpolation", IEEE Trans. on Consumer Electronics, vol.54, no.4, pp.2045-2052, November 2008

[31] S. T. Worrall, A. H. Sadka, P. Sweeney and A. M. Kondoz, "Motion adaptive intra refresh for MPEG-4", Electronics Letters, Vol. 36, No. 23, pp. 1924-1925, November 2000.

[32] SUIT Project, http://suit.av.it.pt/index.htm, Last visited: June 2008.

[33] H. Abdul Karim, "Multiple description coding for 3D video", Phd Thesis, University of Surrey, June 2008.

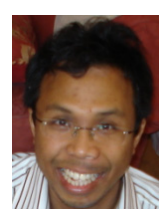

H. Abdul Karim received B.Eng. degree in Electronics with Communications from University of Wales Swansea, UK, in 1998. He received his M. Eng Science degree from Multimedia University, Malaysia in 2003. $\mathrm{He}$ is a Lecturer at Multimedia university since 2002. He obtained his Ph.D degree from Center for Communication Systems Research (CCSR), University of Surrey, UK in 2008. His research interests include telemetry, 2D/3D image/video coding and transmission, error resilience and multiple description video coding.

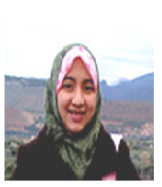

Aduwati Sali received B.Eng. (Hons.) degree in Electrical and Electronics (Communications) Engineering from the University of Edinburgh, UK in 1999. She obtained her MSc from Universiti Putra Malaysia in 2002 and her $\mathrm{PhD}$ degree from CCSR, at the University of Surrey, UK in 2009. She is also a Lecturer at Universiti Putra Malaysia since 2003. Her research interests include radio resource management, cross-layer approaches, reliable multicast, WLAN, WiMax and GEO satellite networks.

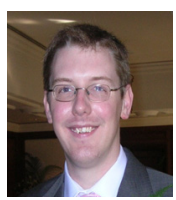

S. Worrall received MEng Electronic Engineering degree from the University of Nottingham in 1998. He joined the CCSR, at the University of Surrey, UK, in 1998 as a research student, and obtained his PhD in 2001. From 2001-2003, he continued to work within CCSR as a research fellow, and was appointed as a lecturer in multimedia communications in 2003. His research interests include error robust video coding, multiple description coding, video compression, transmission of video over wireless networks, and multi-view video coding

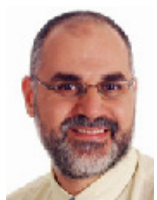

Abdul H. Sadka (Senior Member IEEE) received the B.Eng. degree in computer and communications engineering, the M.Sc. degree in computer engineering, and the Ph.D. degree in electrical and engineering, in 1990, 1993, and 1997, respectively. Professor A. H. Sadka is the head of Electronic and Computer Engineering and the director of the centre for Media Communications Research in Brunel University, UK, with almost 15-year experience in academic leadership and excellence. $\mathrm{He}$ is an internationally renowned expert in visual media processing and communications with an extensive track record of scientific achievements and peer recognised research excellence. He has managed so far to attract over £2M worth of research grants and contracts in his capacity as principal investigator. He has published widely in international journals and conferences and is the author of a highly regarded book on "Compressed Video Communications" published by Wiley in 2002. He holds 3 patents in the video transport and compression area. He acts as scientific advisor and consultant to several key companies in the international Telecommunications sector and is the founder and managing director of VIDCOM Ltd.

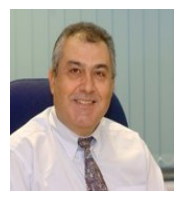

A.M. Kondoz received the B.Sc. (Hons.) degree in engineering, the M.Sc. degree in telematics, and the Ph.D. in communication in 1983, 1984, and 1986, respectively. He became a Lecturer in 1988, a Reader in 1995, and then in 1996, a Professor in Multimedia Communication Systems and deputy director of CCSR, University of Surrey, Guildford, U.K. He has over 250 publications, including a book on low bit-rate speech coding and several book chapters. He has graduated more than $40 \mathrm{Ph}$.D. students in the areas of speech/image and signal processing and wireless multimedia communications, and has been a consultant for major wireless media terminal developers and manufacturers. Prof Kondoz has been awarded several prizes, the most significant of which are The Royal Television Societies' Communications Innovation Award and The IEE Benefactors Premium Award. He has been on the Refereeing College for EPSRC and on the Canadian Research Councils. $\mathrm{He}$ is a member of the IEEE and the IET.B.Eng. 